\title{
Sitagliptin Phosphate: Development of a Dissolution Method for Coated Tablets Based on In Vivo Data for Improving Medium Sensitivity
}

\author{
Alini Dall Cortivo Lange*, Ana Paula Batistel, \\ Letícia Lenz Sfair, Jaison Carlosso, Nadia Maria Volpato, \\ and Elfrides Eva Scherman Schapoval \\ Programa de Pós-Graduação em Ciências Farmacêuticas, Faculdade de Farmácia, Universidade Federal do Rio Grande do Sul. Av. \\ Ipiranga 2752, Lab. 402, Porto Alegre/RS, CEP 90610-000, Brazil
}

\begin{abstract}
Sitagliptin phosphate is a drug used to treat diabetes mellitus type 2 , and it belongs to a new therapeutic class called dipeptidyl peptidase IV inhibitors. This hypoglycemic drug is commercially available in coated tablets containing 25,50, and $100 \mathrm{mg}$ of sitagliptin base. The purpose of this study was to develop and validate the conditions for the dissolution test by investigating a possible in vivo-in vitro correlation. Several parameters were tested to develop the method, and the following conditions were considered satisfactory: $\mathrm{pH} 6.8$ phosphate buffer, $900 \mathrm{~mL}$ of dissolution medium, temperature at $37 \pm 1{ }^{\circ} \mathrm{C}$, paddle apparatus, and rotation speed at $50 \mathrm{rpm}$. The dissolved percentage of STG was quantified by high performance liquid chromatography. The sink condition and specificity were determined in all media tested during method development. The parameters evaluated to validate the method were specificity, linearity, precision, and accuracy. The stability of the sample in phosphate buffer solutions for $24 \mathrm{~h}$ was also determined. The method is linear in the range of $10.0-70.0 \mu \mathrm{g} / \mathrm{mL}$, precise, with RSD values less than $2 \%$, and accurate (mean recovery 98.51\%). The dissolution method as developed and validated supplied a good IVIVC when employing pH 6.8 phosphate buffer medium, which can be used in quality control of sitagliptin coated tablets since no official method has been described.
\end{abstract}

KEYWORDS: In vivo-in vitro correlation; dissolution method; sitagliptin phosphate.

\section{INTRODUCTION}

D iabetes mellitus (DM) presents as a chronic disease in which the pancreas does not produce enough insulin or occurs when the body cannot effectively use the insulin produced. This pathology reduces the quality of life of people living mainly in low- and middleincome countries (1).

Sitagliptin phosphate (STG) (Figure 1) is used to treat DM type 2 because it improves glycemic control by increasing the levels of active incretin hormones, GLP-1 (peptide-1) and GIP (glucose-dependent insulinotropic peptide). The activation of these incretins in $\beta$-pancreatic cells causes increased levels of cyclic adenosine monophosphate (CAMP) and intracellular calcium, with subsequent glucose-dependent insulin secretion (2). This hypoglycemic drug belongs to a new class called dipeptidyl peptidase IV inhibitors (3). STG was approved by the FDA in $2006(4,5)$.

The dissolution test of drugs has been employed as an excellent tool to detect formulation problems that could change drug release in the body (6). The quality of oral solid dosage forms depends on their ability to release the active components in aqueous medium in a consistent

${ }^{*}$ Corresponding author.

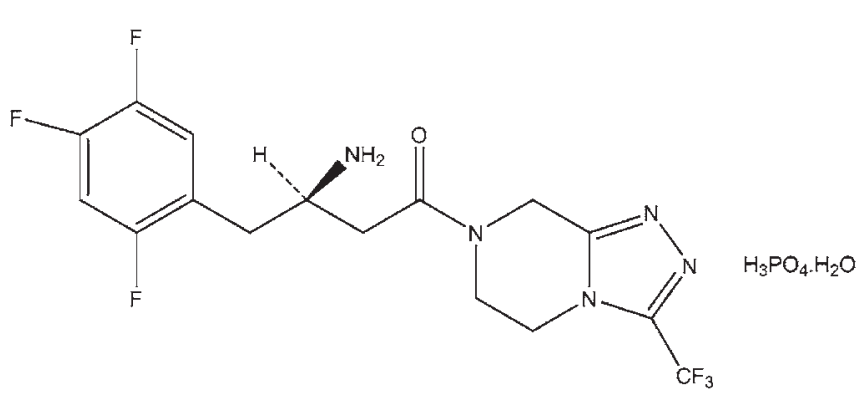

Figure 1. Chemical structure of sitagliptin phosphate.

and reproducible manner, making the active substances available for gastrointestinal absorption (7).

Thus, in vitro dissolution specifications are established to indicate potential problems of bioavailability (6). To characterize a pharmaceutical product, it is necessary to compare the dissolution profiles obtained from different formulations and in diverse conditions. It is thus possible to correlate them with the known aspects of drug bioavailability (8).

The dissolution test is seen as an indispensable tool for the pharmaceutical industry, both in product development and in routine quality control (9-11). 
The solubility and permeability of a drug are considered key parameters for absorption (12). The Biopharmaceutics Classification System (BCS) based on these concepts divides drugs into four categories: Class 1, 2, 3, and 4 (13-15). The use of the BCS determines the possibility of in vivo-in vitro correlation (16).

The FDA has described conditions used for the dissolution test in the STG registration process (dissolution medium: water at $50 \mathrm{rpm}$ ) without an indication of the method used for quantification. Nevertheless, no monograph has been described for sitagliptin tablets. Given the above, the purpose of this study was to develop a method based on in vivo data, which were obtained from the literature (17).

\section{MATERIAL AND METHOD \\ Materials and Reagents}

Sitagliptin phosphate reference standard, $99.5 \%$ purity, was supplied by Sequoia Research Products (Oxford, UK). The coated tablets (Januvia) used in the dissolution test, which contained $50 \mathrm{mg}$ of sitagliptin base, were obtained commercially. The coated tablets contained the inactive ingredients microcrystalline cellulose, calcium phosphate dibasic anhydrous, croscarmellose sodium, magnesium stearate, sodium stearyl fumarate, polyvinyl alcohol, polyethylene glycol, talc, titanium dioxide, yellow ferric oxide, and red ferric oxide, and they were obtained from several distributors. All other chemical reagents were analytical or HPLC grade.

\section{Instrumentation}

The dissolution test was performed using a Vankel VK 7010 multibath $(n=8)$ dissolution testing station attached to a bidirectional peristaltic pump and VK 8000 autosampler.

The quantification of the STG was performed in a highperformance liquid chromatograph (HPLC) Shimadzu 20-A system equipped with a CBM-20A system controller, LC20AT pump, SIL-20A/C auto sampler, CTO-20A/C column oven, and SPD-M2OA PDA detector (Kyoto, Japan). The LC-Solution manager system software was used to control the equipment and to calculate data and responses from the HPLC system. The experiments were performed on a Waters-XBridge C8 (250 mm $\times 5 \mu \mathrm{m}$ ) analytical column (Milford, MA). The HPLC system was operated isocratically at $25^{\circ}$ using a mobile phase composed of a solution of $0.3 \%$ triethylamine adjusted to $\mathrm{pH} 4.0$ with phosphoric acid and acetonitrile $(75 / 25, \mathrm{v} / \mathrm{v})$ at a flow rate of $1.0 \mathrm{~mL} /$ min, using detection at $207 \mathrm{~nm}$. The injection volume was $20 \mu \mathrm{L}$.

\section{Standard Solution Preparation}

The standard solution was prepared in $\mathrm{pH} 6.8$ phosphate buffer using an amount of STG equivalent to $10 \mathrm{mg}$ STG base. The concentration of stock solution was $1.0 \mathrm{mg} /$ $\mathrm{mL}$. This solution was diluted with $\mathrm{pH} 6.8$ phosphate buffer and filtered through a $0.45-\mu \mathrm{m}$ membrane (Millipore) before injection into the analytical column.

\section{Dissolution Test Conditions}

Sitagliptin sink conditions were determined in all media used in the study (9). The solubility of the drug was tested using an amount of solute (STG) and solvent (dissolution medium) equivalent to three times the formulation dose in $900 \mathrm{~mL}$ of medium (10). The media 0.1 M HCl, 0.01 M $\mathrm{HCl}$, water, $\mathrm{pH} 6.8$ phosphate buffer, and $\mathrm{pH} 4.5$ and 5.0 acetate buffer were tested. These media were used because they are relevant to physiological $\mathrm{pH}$ and are frequently used in dissolution testing $(10,14)$.

The dissolution tests were conducted using $900 \mathrm{~mL}$ of each medium. The media were heated and kept at a temperature of $37^{\circ} \mathrm{C}$. USP Apparatus 2 (paddle) at 50 and 75 rpm was tested, and aliquots of $5 \mathrm{~mL}$ were withdrawn at 5 , $10,15,30,45,60,75,90,105$, and $120 \mathrm{~min}$.

\section{In Vivo-In Vitro Correlation (IVIVC)}

The correlation between in vitro and in vivo data for STG was checked by plotting the percentage of STG absorbed (fraction absorbed, FA) versus the average percentage of drug dissolved (fraction dissolved, FD). Linear regression analysis was used to evaluate the data obtained $(18,19)$.

\section{In Vivo Data}

Data for plasma concentration of STG versus time (48 h) were obtained from the literature (17), and it was possible to verify that the drug complies with the one-compartment pharmacokinetic model. The curve of plasma concentration versus time was fitted using the software Micromath Scientist, v. 1.2, to estimate the plasma concentration of STG at the same time as the dissolution evaluation. FA was calculated using the Wagner-Nelson method.

\section{Method Validation}

The dissolution test was validated according to USP and ICH monographs for STG phosphate in coated tablets. The parameters evaluated were specificity, linearity, accuracy, and precision. The stability and system suitability were monitored, and parameters such as theoretical plates, retention time, capacity factor, and symmetry were controlled.

Specificity

A simulated mixture of the formulation excipients was prepared and employed in the specificity evaluation of the method. An amount of mixture equivalent to an average weight of STG was transferred to USP Apparatus 2 vessels $(n=2)$ with $900 \mathrm{~mL}$ of $\mathrm{pH} 6.8$ phosphate buffer medium at $37 \pm 1^{\circ} \mathrm{C}$. After $1 \mathrm{~h}$ at $150 \mathrm{rpm}, 5-\mathrm{mL}$ aliquots were removed, filtered through a $0.45-\mu \mathrm{m}$ membrane, and analyzed by HPLC.

\section{Linearity \\ Method linearity, determined by HPLC, was established by constructing three calibration curves. Aliquots of the}


$1.0 \mathrm{mg} / \mathrm{mL}$ STG standard solution were removed and diluted with $\mathrm{pH} 6.8$ phosphate buffer for concentrations of $10.0,20.0,30.0,40.0,50.0,60.0$, and $70.0 \mu \mathrm{g} / \mathrm{mL}$. This range includes the low concentrations found in the initial STG dissolution time points and exceeds the maximum dose that can be dissolved. The levels were prepared in triplicate, and three determinations were performed for each solution.

\section{Accuracy}

The accuracy was determined by adding known amounts of a powdered STG tablets pool corresponding to drug levels of 25,100 , and $150 \%$ of the labeled amount in an average weight equivalent to a tablet. The dissolution test was run for 30 min using $900 \mathrm{~mL}$ of the medium and Apparatus 2 at $50 \mathrm{rpm}$. This approach was used because of the limited availability of the sitagliptin phosphate reference standard.

\section{Precision}

Repeatability and intermediate precision were used to assess method precision. Repeatability was evaluated through the relative standard deviation (RSD) from adding known amounts of the powdered STG tablets pool, corresponding to a drug level of $100 \%$ (labeled amount in an average weight equivalent to a tablet). The dissolution test was run for 30 min using $900 \mathrm{~mL}$ of medium and Apparatus 2 at 50 rpm, over two days $(n=3)$.

\section{RESULTS AND DISCUSSION}

During the dissolution test, several conditions were tested and adjusted to find a condition that would provide a release of STG from the coated tablets that could be correlated with FA obtained from in vivo data.

Although no biopharmaceutics classification of STG was found, it can be inferred that the drug is Class 1, since it presents high solubility and high permeability (bioavailability of $87 \%$ ). Hence, the absorption process would not be controlled by the solubility and or by permeability. Based on the biopharmaceutics classification, the possibility of in vivo-in vitro correlation is low if the formulation allows rapid drug dissolution, and therefore the rate of absorption is controlled by gastric emptying $(13,20)$. However, the dissolution rate can be significantly altered when the drug is mixed with excipients during production, and in some cases, this can lead to a reduction in bioavailability and clinical response (21). For example, magnesium stearate, which is present in the formulation of STG and is a hydrophobic lubricant, can slow the rate of dissolution of some drugs by reducing the drug-solvent interfacial area (21). These changes in surface characteristics of the tablets result in a decrease in wettability, prolong the disintegration time, and decreasethe interface between the active and the solvent (21).

Nonetheless, the STG tablets have some other components that may delay drug release from the dosage form.
This justifies attempting a possible correlation between in vitro dissolution and in vivo data.

The release rate of STG from the pharmaceutical formulation is $\mathrm{pH}$ dependent. It was observed that the release is faster in acid $\mathrm{pH}$ and slower in neutral $\mathrm{pH}$. The agitation speed of the paddle is a very important feature in the development of the dissolution method. The apparatus routinely used for capsules is the basket, while the paddle is frequently used for tablets with a rotational speed ranging from 50 to $100 \mathrm{rpm}$. The increase from 50 to $75 \mathrm{rpm}$ caused a rapid release of the STG from the pharmaceutical dosage form (22).

The dissolution profiles obtained in different media with paddles at $50 \mathrm{rpm}$ are shown in Figure 2. All tested media reached the sink condition. The $\mathrm{pH} 6.8$ phosphate buffer was the medium that released the drug more slowly, whereas the $0.1 \mathrm{M} \mathrm{HCl}$ medium provided a faster drug release profile.

Despite its designation as BCS Class 1, STG did not dissolve rapidly from the tablets in all media evaluated. If the excipients play a role in STG dissolution, as observed in vitro, media employing water and $\mathrm{pH} 6.8$ phosphate buffer could show this slower release. On the other hand, if rapid dissolution of this hypoglycemic agent had been obtained at all $\mathrm{pH}$ levels evaluated, the drug could be a candidate for a biowaiver approach in the development of a generic product. As this situation was not achieved, medium that is more sensitive to characterize the formulation can be used more efficaciously in quality control of the tablets and probably in the development of a new product.

The HPLC method had been developed and validated for quantitative analysis of the STG in tablets. However, some changes were necessary for application to the dissolution test, for example, adjustments in the range of linearity and the solvent used for sample dilution. Due to the low concentration of STG in the dissolution medium,

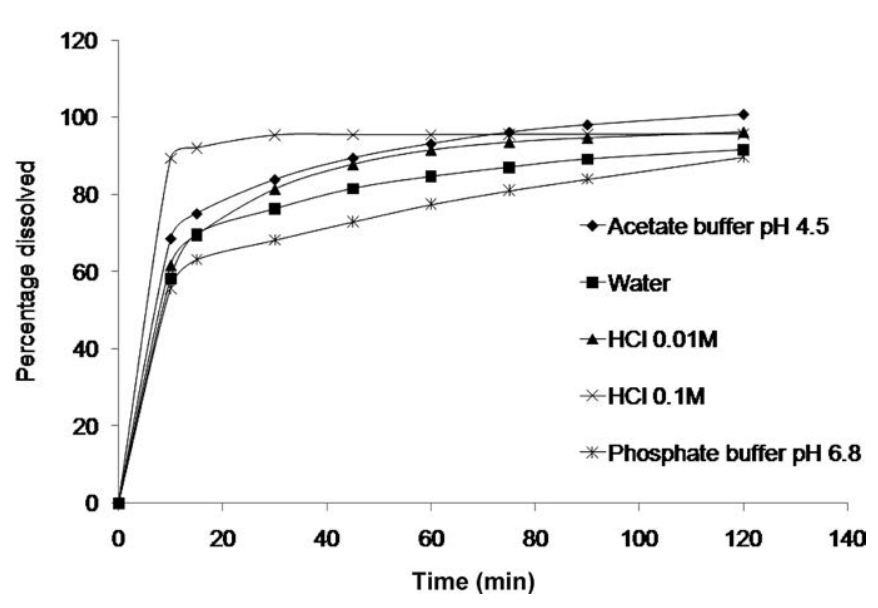

Figure 2. Dissolution profiles of sitagliptin phosphate coated tablets $(n=6)$ employing pH 4.5 acetate buffer, water, $0.01 \mathrm{M} \mathrm{HCl}, 0.1 \mathrm{M} \mathrm{HCl}$, and pH 6.8 phosphate buffer at $50 \mathrm{rpm}$. 
it was not possible to perform a second dilution with the mobile phase. The samples were collected, filtered, and analyzed directly.

The linearity was evaluated in the concentration range of $10-70 \mu \mathrm{g} / \mathrm{mL}$. The representative linear equation for STG was $y=42031 x+27210$, with a determination coefficient of 0.9995 . The data were validated by analysis of variance (ANOVA), which showed significant linear regression and non-significant linearity deviation $\left(F_{\text {calc }}=0.15<\right.$ $\left.F_{\text {crit }}=4.53 ; \alpha=0.05\right)$.

The specificity of the method was evaluated to verify that the formulation excipients and the dissolution medium did not interfere in the analysis of STG. Figure 3 represents the chromatogram obtained for the solutions of the tablets and the formulation excipients. No interference of the formulation excipients was observed in the analysis of STG by HPLC method, as there is no peak at the same retention time as that of STG. The peak purity was determined by Shimadzu LC-Solution software tools.

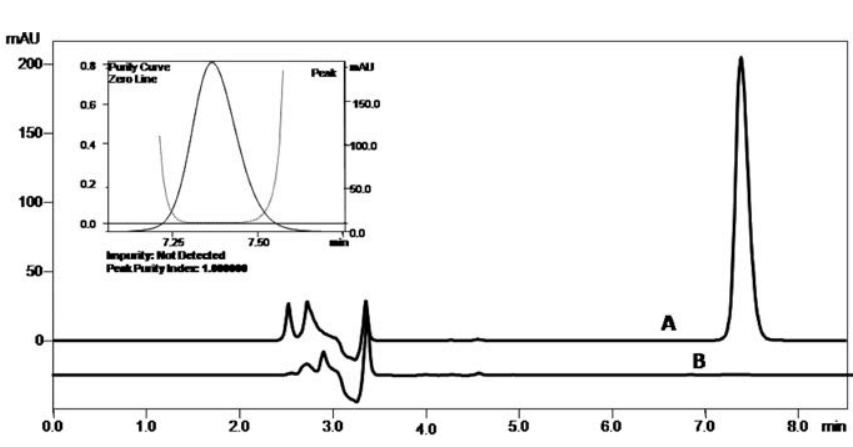

Figure 3. Overlay of chromatograms of (A) STG coated tablets and (B) excipients simulated sample by HPLC.

Table 1. Intraday and Interday Precision Results for STG Dissolved in $900 \mathrm{~mL}$ of Medium and Analyzed by LC

\begin{tabular}{lll}
\hline \multicolumn{1}{c}{ Sample } & Day 1 & Day 2 \\
\hline $\mathbf{1}$ & 99.74 & 98.18 \\
\hline $\mathbf{2}$ & 99.33 & 98.17 \\
\hline $\mathbf{3}$ & 98.77 & 99.36 \\
\hline Average content (\%) & 99.28 & 98.57 \\
\hline Intraday RSD (\%) & 0.49 & 0.69 \\
\hline Interday RSD (\%) & & 0.66 \\
\hline
\end{tabular}

Method precision and accuracy were adjusted and redesigned for the execution of validation parameters. Thus, a pool of STG coated tables was prepared, finely triturated, and used for analysis. The pool content was checked every day against a standard solution with known concentration of STG to evaluate precision and accuracy.

The results obtained for the precision of the method are described in Table 1. The values of relative standard deviation (RSD) were less than $2.0 \%$ for repeatability and intermediate precision, demonstrating good precision of the dissolution procedure.

The accuracy of the method was demonstrated by the recovery of the known amounts of sitagliptin phosphate added from the tablet pool to the dissolution vessels. Three levels were evaluated, low, medium, and high. The results obtained are described in Table 2 . The percentage recoveries ranging from $98 \%$ to $102 \%$ demonstrate the satisfactory accuracy of the method.

To select the medium (water or pH 6.8 phosphate buffer) that could better correlate with in vivo data, a level A correlation study was attempted.

From the plasma concentration-versus-time data obtained in the literature (17) and knowledge that the drug follows the one-compartment pharmacokinetics model, the Wagner-Nelson approach was applied, and the FA was calculated. To infer the FA from the exact times used in development of the dissolution profile, the Scientist program was used.

The FA for the times 10, 20, 30, 45, 60, 75, 90, 105, and 120 min was determined. Hereafter, the FA was correlated with the FD (data obtained in the dissolution test). As mentioned previously, Class 1 drugs are less likely to achieve a perfect correlation employing the same times for in vivo and in vitro data. The correlation demonstrated in Figure 4 is not ideal; although the correlation coefficient is closer to 1 ( $r=0.9929)$, the slope is greater than 1 . In other words, the relationship confirms a faster in vitro dissolution as compared with the FA. The adoption of a timescale factor could improve the slope of the IVIVC, as the $x$-axis could be stretched by a factor of 3 or 4, for example. However, the introduction of this approach implies, per se, to work with a faster in vitro dissolution profile than in vivo absorption profile.

The correlation between STG FA and FD was tested in all media used in the study ( $\mathrm{pH} 4.5$ acetate buffer, water, $0.01 \mathrm{M} \mathrm{HCl}, 0.1 \mathrm{M} \mathrm{HCl}$, and $\mathrm{pH} 6.8$ phosphate buffer). Water as a dissolution medium showed good results but was not as satisfactory as $\mathrm{pH} 6.8$ buffer phosphate. Although

Table 2. Mean Recovery of STG Added by Means of the Powdered Tablets Pool

\begin{tabular}{cccc}
\hline Level (\%) & Added STG $(\mathbf{m g})$ & Added tablets "pool" $(\mathbf{m g})$ & Mean recovery \pm RSD $(\%)$ \\
\hline $\mathbf{2 5}$ & 12.5 & 51.75 & $98.99 \pm 1.80$ \\
\hline $\mathbf{1 0 0}$ & 50.0 & 207.0 & $98.73 \pm 0.68$ \\
\hline $\mathbf{1 5 0}$ & 75.0 & 310.5 & $97.81 \pm 0.76$ \\
\hline
\end{tabular}




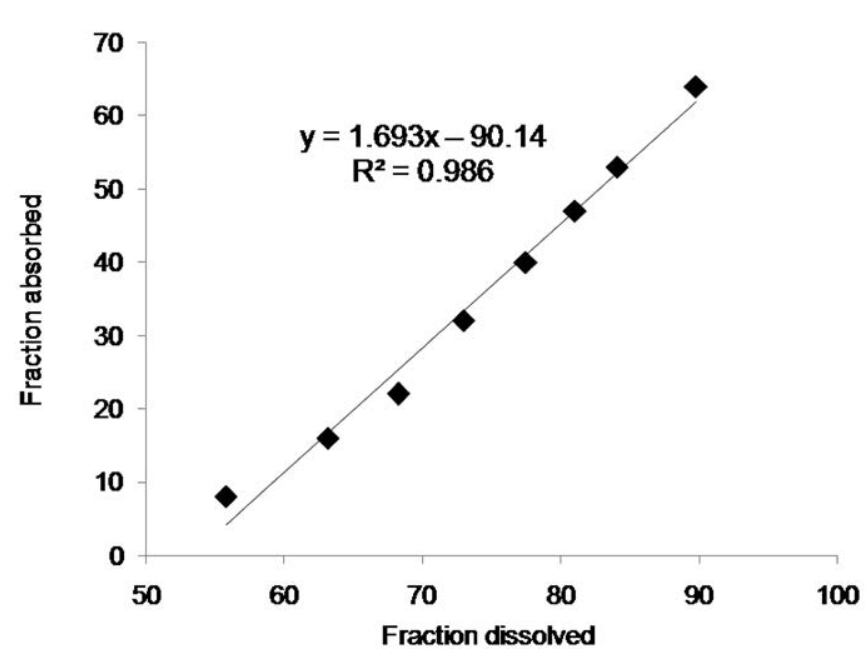

Figure 4. Plot of fraction of dose absorbed (FA) in vivo versus fraction of dose dissolved (FD) in vitro for STG phosphate coated tablets.

water is the dissolution medium registered at the FDA for STG, it was not chosen because it presents variable $\mathrm{pH}$ values and therefore its use has been discouraged.

\section{CONCLUSIONS}

Validation of the dissolution test results shows that the method is specific, linear, precise, and accurate. Both the HPLC analytical method and the in vitro dissolution test were validated and can be used to evaluate the release profile of sitagliptin phosphate from coated tablets. The $\mathrm{pH} 6.8$ phosphate buffer with Apparatus 2 at $50 \mathrm{rpm}$ allowed a slow release of sitagliptin from the pharmaceutical formulation, thus making it possible to correlate the data of the FA with those of the FD. Moreover, a slower dissolution profile usually presents higher discriminative power for use as a control quality tool for formulations and processes.

\section{ACKNOWLEDGMENTS}

The authors thank the National Council of Scientific and Technological Development (CNPq) for the financial support.

\section{REFERENCES}

1. World Health Organization. Global Prevalence of Diabetes: Estimates for the year 2000 and projections for 2030. http://www.who.int/diabetes/facts/en/diabcare0504.pdf (accessed April 13, 2014).

2. Drucker, D. J.; Nauck, M. A. The incretin system: glucagon-like peptide-1 receptor agonists and dipeptidyl peptidase-4 inhibitors in type 2 diabetes. Lancet 2006, 368 (9548), 1696-1705. DOI: 10.1016/S01406736(06)69705-5.

3. Ahrén, B. DPP-4 inhibitors. Best Pract. Res., Clin. Endocrinol. Metab. 2007, 21 (4), 517-533. DOI: 10.1016/j.beem.2007.07.005.
4. Zerilli, T.; Pyon, E. Y. Sitagliptin phosphate: A DPP-4 inhibitor for the treatment of type 2 diabetes mellitus. Clin. Ther. 2007, 29 (12), 2614-2634.

5. JANUMET. Circular aos mêdicos. Laboratório Merck Sharp \& Dohme: São Paulo, 2008.

6. Anvisa. Resolução-RDC No. 31. Bioavailability and bioequivalence studies; Guidance for Industry; ICF Ciências Farmacêuticas, Brazil, 2010.

7. Davydova, N.; Stippler, E.; Jin, P.; Giancaspro, G. Development and validation of a dissolution test method for vitamin A in dietary supplement tablets. J. Pharm. Biomed. Anal. 2010, 53 (3), 295-301. DOI: 10.1016/j.jpba.2010.03.036.

8. Yuksel, N.; Kanik, A. E.; Baykara, T. Comparison of in vitro dissolution profiles by ANOVA-based, modeldependent and -independents methods. Int. J. Pharm. 2000, 209 (1-2), 57-67. DOI: 10.1016/S03785173(00)00554-8.

9. Garcia, C. V.; Paim, C. S.; Steppe, M.; Schapoval, E. E. $S$. Development and validation of a dissolution test for rabeprazole sodium in coated tablets. J. Pharm. Biomed. Anal. 2006, 41 (3), 833-837. DOI: 10.1016/j. jpba.2006.01.050.

10. Marques, M. R. C.; Brown, W. Desenvolvimento e validação de métodos de dissolução para formas farmacêuticas sólidas orais. Revista Analytica 2002, 1, 48-51.

11. Marcolongo, R. Dissertação de mestrado, Universidade de São Paulo, São Paulo, Brasil, 2003. Dissolução de medicamentos: fundamentos, aplicações, aspectos regulatórios e perspectivas na área farmacêutica.

12. Löbenberg, R.; Krämer, J.; Shah, V. P.; Amidon, G. L.; Dressman, J. B. Dissolution Testing as a Prognostic Tool for Oral Drug Absorption: Dissolution Behavior of Glibenclamide. Pharm. Res. 2000, 17 (4), 439-444. DOI: 10.1023/A:1007529020774.

13. Amidon, G. L.; Lennernäs, H.; Shah, V. P.; Crison, J. R. A Theoretical Basis for a Biopharmaceutic Drug Classification:The Correlation of in Vitro Drug Product Dissolution and in Vivo Biovailability. Pharm. Res. 1995, 12 (3), 413-420. DOI: 10.1023/A:1016212804288.

14. Dissolution Testing of Immediate Release Solid Oral Dosage Forms; Guidance for Industry; U.S. Department of Health and Human Services, Food and Drug Administration, Center for Drug Evaluation and Research (CDER), U.S. Government Printing Office: Washington, DC, 1997.

15. Waiver of In Vivo Bioavailability and Bioequivalence Studies for Immediate-Release Solid Oral Dosage Forms Based on a Biopharmaceutics Classification System; Guidance for Industry; U.S. Department of Health and Human Services, Food and Drug Administration, Center for Drug Evaluation and Research (CDER), U.S. Government Printing Office: Washington, DC, 2000.

16. Brown, C. K.; Chokshi, H. P.; Nickerson, B.; Reed, R. A.; Rohrs, B. R.; Shah, P. A. Acceptable Analytical Practices 
for Dissolution Testing of Poorly Soluble Compounds. Pharm. Technol. 2004, 28 (12), 56-65.

17. Bergman, A.; Ebel, D.; Liu, F.; Stone, J.; Wang, A.; Zeng, W.; Chen, L.; Dilzer, S.; Lasseter, K.; Herman, G.; Wagner, J.; Krishna, R. Absolute bioavailability of sitagliptin, an oral dipeptidyl peptidase-4 inhibitor, in healthy volunteers. Biopharm. Drug Dispos. 2007, 28 (6), 315-322. DOI: $10.1002 /$ bdd.560.

18. Pharmaceutical Product Development: In Vitro-In Vivo Correlation; Chilukuri, D. M., Sunkara, G., Young, D., Eds.; Drugs and the Pharmaceutical Sciences, Vol. 165; Informa Healthcare USA, Inc.: New York, 2007.

19. Rossi, R. C.; Dias, C. L.; Bajerski, L.; Bergold, A. M.; Fröehlich, P. E. Development and validation of discriminating method of dissolution for fosam- prenavir tablets based on in vivo data. J. Pharm. Biomed. Anal. 2011, 54 (3), 439-444. DOI: 10.1016/j. jpba.2010.09.004.

20. Galia, E.; Nicolaides, E.; Hörter, D.; Löbenberg, R.; Reppas, C.; Dressman, J.B. Evaluation of Various Dissolution Media for Predicting In Vivo Performance of Class I and II Drugs. Pharm. Res. 1998, 15 (5), 698705. DOI: 10.1023/A:1011910801212.

21. Abdou, H. M. Dissolution. In: Remington: The Science and Practice of Pharmacy, 19th ed.; Gennaro, A., Ed.; Lippincott Williams \& Wilkins: Philadelphia, 1995; pp 593-604.

22. The United States Pharmacopeia and National Formulary USP 34-NF 29; The United States Pharmacopeial Convention, Inc.: Rockville, MD, 2011. 